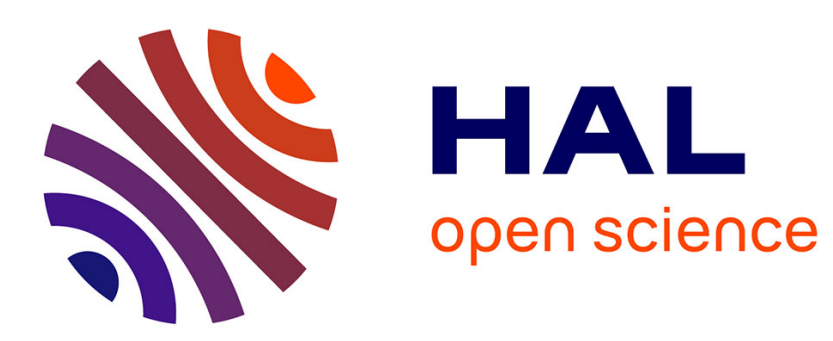

\title{
The Kolmogorov Law of turbulence, What can rigorously be proved? Part II
}

Roger Lewandowski, Benoît Pinier

\section{To cite this version:}

Roger Lewandowski, Benoît Pinier. The Kolmogorov Law of turbulence, What can rigorously be proved ? Part II. The Foundations of chaos revisited: from Poincaré to recent advancements, Springer, pp.71-89, 2016, 978-3-319-29699-9. 10.1007/978-3-319-29701-9_5. hal-01244651

\section{HAL Id: hal-01244651 \\ https://hal.science/hal-01244651}

Submitted on 18 Jan 2016

HAL is a multi-disciplinary open access archive for the deposit and dissemination of scientific research documents, whether they are published or not. The documents may come from teaching and research institutions in France or abroad, or from public or private research centers.
L'archive ouverte pluridisciplinaire HAL, est destinée au dépôt et à la diffusion de documents scientifiques de niveau recherche, publiés ou non, émanant des établissements d'enseignement et de recherche français ou étrangers, des laboratoires publics ou privés. 


\title{
The Kolmogorov Law of turbulence What can rigorously be proved? Part II
}

\author{
Roger Lewandowski and Benoît Pinier*
}

\begin{abstract}
We recall what are the different known solutions for the incompressible NavierStokes Equations, in order to fix a suitable functional setting for the probabilistic frame that we use to derive turbulence models, in particular to define the mean velocity and pressure fields, the Reynolds stress and eddy viscosities. Homogeneity and isotropy are discussed within this framework and we give a mathematical proof of the famous $-5 / 3$ Kolmogorov law, which is discussed in a numerical simulation performed in a numerical box with a non trivial topography on the ground.
\end{abstract}

MCS Classification : 76D05, 76F65, 65M60,

\section{Contents}

1 Introduction $\quad 2$

2 About the 3D Navier Stokes equations 3

2.1 Framework . . . . . . . . . . . . . . . . . 3

2.2 Strong solutions to the NSE . . . . . . . . . . . . . . . 4

2.3 Turbulent solutions . . . . . . . . . . . . . . . . . . . . 5

3 Mean Navier-Stokes Equations

3.1 Reynolds decomposition . . . . . . . . . . . . . . . . . . 5

3.2 Reynolds Stress and closure equations . . . . . . . . . . . . . . 6

4 Law of the $-5 / 3 \quad 7$

4.1 Energy density of the flow . . . . . . . . . . . . . . . 7

4.2 Dimensional bases . . . . . . . . . . . . . . . . . . . . . . . . 9

4.3 Kolmogorov scales . . . . . . . . . . . . . . . . . . 9

4.4 Proof of the $-5 / 3$ 's law . . . . . . . . . . . . . 9

5 Numerical experiments $\quad 11$

5.1 Simulation setting . . . . . . . . . . . . . . . . . . . . 11

5.2 Results . . . . . . . . . . . . . . . . . . . . . . . 12

${ }^{*}$ IRMAR, UMR 6625, Université Rennes 1, and Fluminance Team INRIA Campus Beaulieu, 35042 Rennes cedex FRANCE; Roger.Lewandowski@univ-rennes1.fr, benoit.pinier@inria.fr, http://perso.univrennes1.fr/roger.lewandowski/ 


\section{Introduction}

We focus in this paper on the law of the $-5 / 3$, which attracted a lot of attention from the fluid mechanics community these last decades, since it is a basis for many turbulence models, such as Large Eddy Simulation models (see for instance in [20, 21, 44, 50]). Although it is usually known as the Kolmogorov law, it seems that it appears for the first time in a paper by Onsager [42] in 1949, and not in the serie of papers published by Kolmogorov in 1941 (see in [56]), where the author focuses on the 2/3's law, by introducing the essential scales related to homogeneous and isotropic turbulent flows (see formula (4.13) below). In this major contribution to the field, Kolmogorov opened the way for the derivation of laws based on similarity principles such as the $-5 / 3$ 's law (see also in $[11,32]$ ).

Roughly speaking, the $-5 / 3$ 's law states that in some inertial range $\left[k_{1}, k_{2}\right]$, the energy density of the flow $E(k)$ behaves like $C^{t e} k^{-5 / 3}$, where $k$ denotes the current wave number (see figure 1 below and the specific law (4.20)).

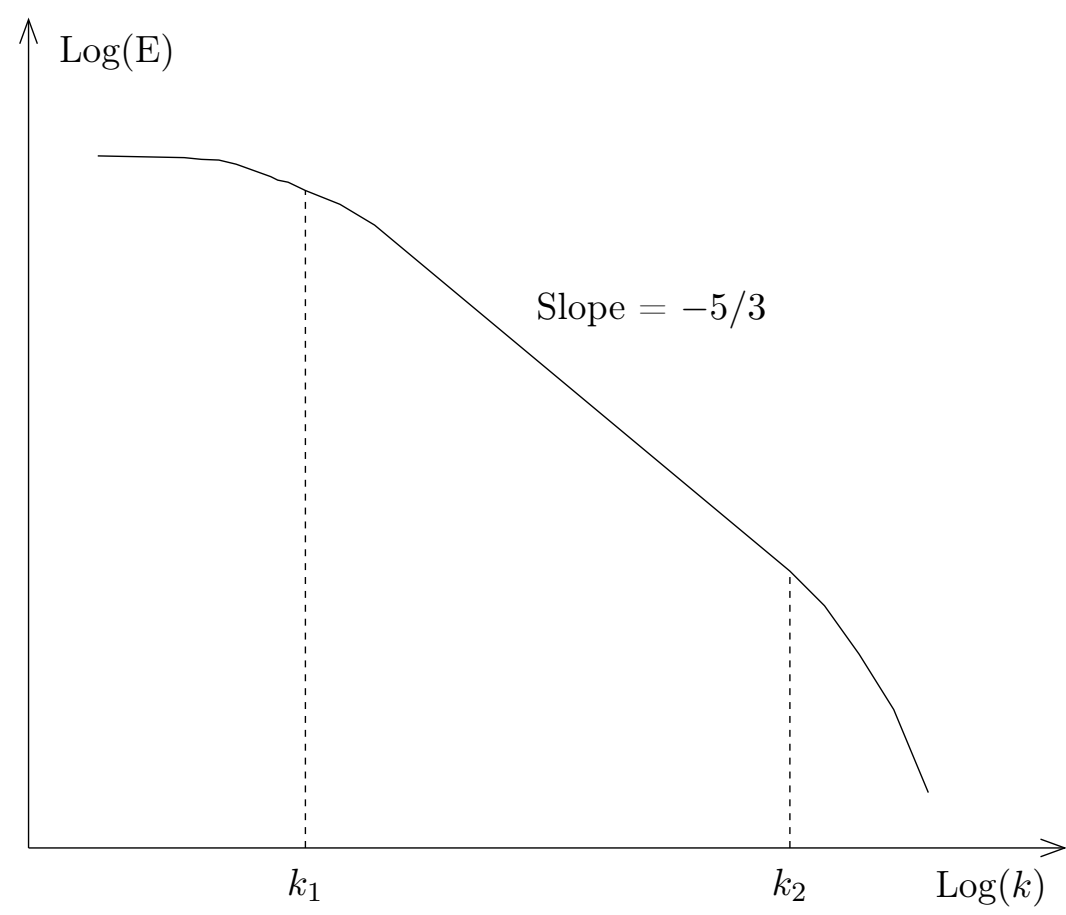

Figure 1: Energy spectrum Log-Log curve

This paper is divided in a theoretical part and a numerical part, in which we aim at:

i) carrefully express what is the appropriate similarity assumption that must satisfy an homogeneous and istropic turbulent flow in order to derive the $-5 / 3$ 's law (assumptions 4.1 and 4.2 below),

ii) to theoretically derive the $-5 / 3$ law from the similarity assumption (see Theorem 4.2 below),

iii) to discuss the numerical validity of such a law from a numerical simulation in a test case, using the software BENFLOW 1.0, developed at the Institute of Mathematical Research of Rennes. 
Before processing items i) and ii), we discuss on different results about the Navier-Stokes equations (2.1) (NSE in what follows), that are one of the main tools in fluid mechanics, as well as the Reynolds stress (3.7) derived by taking the expectation of the NSE, once the appropriate probabilistic frame is specified. We then define the density energy $E(k)$, which is the energy of the flow in the sphere $\{k=|\mathbf{k}|\}$ in the Fourier space. Furthermore, we introduce the concept of dimensional bases in order to properly set Assumptions 4.1 and 4.2 .

The numerical simulation takes place in a computational box with a non trivial topography (see figure 3), by using the mean NSE (3.6), the $k-\mathscr{E}$ model (3.14), and appropriate boundary conditions supposed to model the dynamics of the atmospheric boundary layer. Atmospheric boundary layer modeling is a modern challenge because of its significance in climate change issues. We find in the literature many simulations carried out in different configurations, such as for example the case of a flat ground $[1,5,13,45]$, the case of stable or convective boundary layers $[38,59]$, urban simulations where building are modeled by parallelipipeds [39], wind farms [46], realistic configurations including mountains [37, 58]. Of course, this flows is not homogeneous nor isotropic. However, the simulations shows that the curve of $\log _{10}(E(k))$ exhibits an inertial range over 4 decades, in which the regression straight line has a slope equal to $-2.1424 \neq-5 / 3$ (see figure 6), suggesting that the $-5 / 3$ 's law is not satisfied in this case.

\section{About the 3D Navier Stokes equations}

\section{$2.1 \quad$ Framework}

Let $\Omega \subseteq \mathbb{R}^{3}$ be a $C^{1}$ bounded convex smooth domain, $\Gamma$ its boundary, $T \in \mathbb{R}_{+}$(enventually $T=+\infty)$, and $Q=[0, T] \times \Omega$. The velocity of the flow is denoted by $\mathbf{v}$, its pressure by $p$. The incompressible Navier Stokes equation satisfied by $(\mathbf{v}, p)$ (NSE in the remainder) are as follows:

$$
\left\{\begin{aligned}
\partial_{t} \mathbf{v}+(\mathbf{v} \cdot \nabla) \mathbf{v}-\nabla \cdot(2 \nu D \mathbf{v})+\nabla p & =\mathbf{f} & & \text { in } Q, \\
\nabla \cdot \mathbf{v} & =0 & & \text { in } Q, \\
\mathbf{v} & =0 & & \text { on } \Gamma, \\
\mathbf{v} & =\mathbf{v}_{0} & & \text { at } t=0,
\end{aligned}\right.
$$

where $\mathbf{v}_{0}$ is any divergence free vector fields such that $\left.\mathbf{v}_{0} \cdot \mathbf{n}\right|_{\Gamma}=0, \nu>0$ denotes the kinematic viscosity, that we suppose constant for the simplicity, $\mathbf{f}$ is any external force (such as the gravity for example), $D \mathbf{v}$ denotes the deformation tensor, $\nabla \cdot$ the divergence operator and $(\mathbf{v} \cdot \nabla) \mathbf{v}$ is the nonlinear transport term, specifically

$$
\begin{aligned}
& D \mathbf{v}=\frac{1}{2}\left(\nabla \mathbf{v}+\nabla \mathbf{v}^{t}\right), \quad \nabla \mathbf{v}=\left(\partial_{j} v_{i}\right)_{1 \leq i j \leq 3}, \quad \mathbf{v}=\left(v_{1}, v_{2}, v_{3}\right), \quad \partial_{i}=\frac{\partial}{\partial x_{i}}, \\
& \nabla \cdot \mathbf{v}=\partial_{i} v_{i}, \\
& {[(\mathbf{v} \cdot \nabla) \mathbf{v}]_{i}=v_{j} \partial_{j} v_{i},}
\end{aligned}
$$

by using the Einstein summation convention. We recall that it is easily deduced from the incompressibilty condition (see [11]):

$$
\begin{aligned}
& (\mathbf{v} \cdot \nabla) \mathbf{v}=\nabla \cdot(\mathbf{v} \otimes \mathbf{v}), \quad \mathbf{v} \otimes \mathbf{v}=\left(v_{i} v_{j}\right)_{1 \leq i, j \leq 3}, \\
& \nabla \cdot(2 \nu D \mathbf{v})=\nu \Delta \mathbf{v} .
\end{aligned}
$$

In the following, we will consider the functional spaces

$$
\mathbf{W}=\left\{\mathbf{v} \in H_{0}^{1}(\Omega)^{3}, \nabla \cdot \mathbf{v}=0\right\} \hookrightarrow \mathbf{V}=\left\{\mathbf{v} \in L^{2}(\Omega)^{3},\left.\mathbf{v} \cdot \mathbf{n}\right|_{\Gamma}=0, \nabla \cdot \mathbf{v}=0\right\},
$$


Throughout the paper, we assume $\mathbf{v}_{0} \in \mathbf{V}$.

\subsection{Strong solutions to the NSE}

Let $\mathrm{P}$ be the orthogonal projection $L^{2}(\Omega)^{3} \hookrightarrow \mathbf{V}, A$ and $F$ the operators

$$
A \mathbf{v}=-\nu P \Delta \mathbf{v}, \quad F \mathbf{v}=P((\mathbf{v} \cdot \nabla) \mathbf{v}) .
$$

By applying $P$ to $(2.1 . \mathrm{i})$ in noting that $P(\nabla p)=0$, we are led to the following initial value problem

$$
\left\{\begin{array}{l}
\frac{d \mathbf{v}}{d t}=-A \mathbf{v}+F \mathbf{v}+P \mathbf{f}(t) \\
\mathbf{v}(0)=\mathbf{v}_{0}
\end{array}\right.
$$

where $t \rightarrow \mathbf{v}(t)$ and $t \rightarrow \mathbf{f}(t)$ are considered as functions valued in $\mathbf{W}$ and $\mathbf{V}$ respectively.

Definition 2.1. We say that $\mathbf{v}=\mathbf{v}(t)$ is a strong solution to the NSE in a time interval $\left[0, T^{\star}\right]$ if $d \mathbf{v} / d t$ and $A \mathbf{v}$ exist and are continuous in $\left[0, T^{\star}\right]$ and (2.3.i) is satisfied there.

Remark 2.1. In definition 2.1, the pressure is not involved. It can be reconstructed by the following equation

$$
\Delta p=-\nabla \cdot((\mathbf{v} \cdot \nabla) \mathbf{v})+\nabla \cdot \mathbf{f},
$$

derived from equation (2.1.i) by taking its divergence.

The existence of a strong solution is proved in Fujita-Kato [18]. It is subject to regularity conditions regarding the initial data $\mathbf{v}_{0}$ and the source $\mathbf{f}$. The result is stated as follows.

Theorem 2.1. We assume

i) $\mathbf{v}_{0} \in \mathbf{V} \cap H^{1 / 2}(\Omega)^{3}$,

ii) $\mathbf{f}$ is Hölder continuous in $[0, T]$.

Then there exists $T^{\star}=T^{\star}\left(\nu,\left\|\mathbf{v}_{0}\right\|_{1 / 2,2, \Omega},\|\mathbf{f}\|_{C^{0, \alpha}(\Omega)}\right)$ such that the NSE admits a unique strong solution $\mathbf{v}=\mathbf{v}(t)$. Moreover, if $\mathbf{f}=\mathbf{f}(t, \mathbf{x})$ is Hölder continuous in $Q=\left[0, T^{\star}\right] \times \Omega$, then $\mathbf{v}(t, \mathbf{x}), \nabla \mathbf{v}(t, \mathbf{x}), \Delta \mathbf{v}(t, \mathbf{x})$ and $\partial \mathbf{v}(t, \mathbf{x}) / \partial t$ are Hölder continous in $] 0, T^{\star}[\times \Omega$.

Remark 2.2. The strong solution is solution of the equation

$$
\mathbf{v}(t)=e^{-t A} \mathbf{v}_{0}-\int_{0}^{t} e^{-(t-s) A} F(\mathbf{v}(s)) d s+\int_{0}^{t} e^{-(t-s) A} P \mathbf{f}(s) d s,
$$

which is approached by the sequence $\left(\mathbf{v}_{n}\right)_{n \in \mathbf{N}}$ expressed by

$$
\mathbf{v}_{n}(t)=e^{-t A} \mathbf{v}_{0}-\int_{0}^{t} e^{-(t-s) A} F\left(\mathbf{v}_{n-1}(s)\right) d s+\int_{0}^{t} e^{-(t-s) A} P \mathbf{f}(s) d s,
$$

The reader is referred to $[9,12,28]$ for more details concerning the question of strong solutions. 


\subsection{Turbulent solutions}

Definition 2.2. We say that $\mathbf{v}$ is a turbulent solution of NSE (2.1) in $[0, T]$ if

i) $\mathbf{v} \in L^{2}([0, T], \mathbf{W}) \cap L^{\infty}\left([0, T], L^{2}(\Omega)\right)$,

ii) $\partial_{t} \mathbf{v} \in L^{4 / 3}\left([0, T], \mathbf{W}^{\prime}\right)=\left[L^{4}([0, T], \mathbf{W})\right]^{\prime}$ (by writing $\partial_{t}=\frac{\partial}{\partial t}$ for the simplicity),

iii) $\lim _{t \rightarrow 0}\left\|\mathbf{v}(\cdot, t)-\mathbf{v}_{0}(\cdot)\right\|_{0,2, \Omega}=0$,

iv) $\forall \mathbf{w} \in L^{4}([0, T], \mathbf{W})$,

$\int_{0}^{T}<\partial_{t} \mathbf{v}, \mathbf{w}>d t+\int_{0}^{T} \int_{\Omega}(\mathbf{v} \otimes \mathbf{v}): \nabla \mathbf{w} d \mathbf{x} d t+\int_{0}^{T} \int_{\Omega} \nabla \mathbf{v}: \nabla \mathbf{w} d \mathbf{x} d t=\int_{0}^{T}<\mathbf{f}, \mathbf{w}>d t$,

where for $\mathbf{u} \in \mathbf{W}, \mathbf{F} \in \mathbf{W}^{\prime},<\mathbf{F}, \mathbf{u}>$ denotes the duality pairing between $\mathbf{F}$ and $\mathbf{u}$,

v) $\mathbf{v}$ satisfies the energy inequality at each $t>0$,

$$
\frac{1}{2} \int_{\Omega}|\mathbf{v}(t, \mathbf{x})|^{2} d \mathbf{x}+\nu \int_{0}^{t} \int_{\Omega}\left|\nabla \mathbf{v}\left(t^{\prime}, \mathbf{x}\right)\right|^{2} d \mathbf{x} d t^{\prime} \leq \int_{0}^{t}<\mathbf{f}, \mathbf{v}>d t^{\prime} .
$$

Remark 2.3. Once again, the pressure is not involved in this formulation. It this frame, it is recovered by the De Rham Theorem (see for instance in [55]).

The existence of a turbulent solution was first proved by Leray [29] in the whole space, then by Hopf [22] in the case of a bounded domain with the no slip boundary condition, which is the case under conderation here. This existence result can be stated as follows.

Theorem 2.2. Assume that $\mathbf{v}_{0} \in \mathbf{V}, \mathbf{f} \in L^{4 / 3}\left([0, T], \mathbf{W}^{\prime}\right)$. Then the NSE (2.1) has a turbulent solution.

Remark 2.4. The turbulent solution is global in time, which means that it may be extended to $t \in[0, \infty$ [ depending on a suitable assumption on $\mathbf{f}$. However it is not known whether it is unique or not. Moreover, it is not known if the energy inequality is an equality.

The reader is also referred to $[14,16,36,55]$ for further results on turbulent (also weak) solutions of the NSE.

\section{Mean Navier-Stokes Equations}

\subsection{Reynolds decomposition}

Based on strong or turbulent solutions, it is known that it is possible to set a probabilistic framework in which we can decompose the velocity $\mathbf{v}$ and the pressure as a the sum of the statistical mean and a fluctuation, namely

$$
\mathbf{v}=\overline{\mathbf{v}}+\mathbf{v}^{\prime}, \quad p=\bar{p}+p^{\prime} .
$$

More generally, any tensor field $\psi$ related to the flow can be decomposed as

$$
\psi=\bar{\psi}+\psi^{\prime} .
$$


The statistical filter is linear and subject to satisfy the Reynolds rules:

$$
\begin{aligned}
& \overline{\partial_{t} \psi}=\partial_{t} \bar{\psi}, \\
& \overline{\nabla \psi}=\nabla \bar{\psi},
\end{aligned}
$$

as well as

$$
\overline{\bar{\psi}}=\bar{\psi} \text { leading to } \overline{\psi^{\prime}}=0 .
$$

We have studied in [11] different examples of such filters. Historically, such a decomposition was first considered in works by Stokes [53], Boussinesq [6], Reynolds [49], Prandtl [47], in the case of the « long time average » (see also in [31]). Later on, Taylor [54], Kolmogorov [25] and Onsager [42] have considered such decompositions when the fields related to the flow are considered as random variables, which was one of the starting point for the development of modern probability theory.

\subsection{Reynolds Stress and closure equations}

We take the mean of the NSE (2.1) by using (3.3), (3.4) and (3.5). We find out the following system:

$$
\left\{\begin{aligned}
\partial_{t} \overline{\mathbf{v}}+(\overline{\mathbf{v}} \cdot \nabla) \overline{\mathbf{v}}-\nu \Delta \overline{\mathbf{v}}+\nabla \bar{p} & =-\nabla \cdot \boldsymbol{\sigma}^{(\mathrm{R})}+\mathbf{f} & & \text { in } Q, \\
\nabla \cdot \overline{\mathbf{v}} & =0 & & \text { in } Q, \\
\overline{\mathbf{v}} & =0 & & \text { on } \Gamma, \\
\overline{\mathbf{v}} & =\overline{\mathbf{v}_{0}} & & \text { at } t=0,
\end{aligned}\right.
$$

where

$$
\boldsymbol{\sigma}^{(\mathrm{R})}=\overline{\mathbf{v}^{\prime} \otimes \mathbf{v}^{\prime}}
$$

is the Reynolds stress. The big deal in turbulence modeling is to express $\boldsymbol{\sigma}^{(\mathrm{R})}$ in terms of averaged quantities. The most popular model is derived from the Boussinesq assumption which consists in writing:

$$
\boldsymbol{\sigma}^{(\mathrm{R})}=-\nu_{t} D \overline{\mathbf{v}}+\frac{2}{3} k \mathrm{Id},
$$

where

i) $k=\frac{1}{2} \operatorname{tr} \boldsymbol{\sigma}^{(\mathrm{R})}=\frac{1}{2} \overline{\left|\mathbf{v}^{\prime}\right|^{2}}$ is the turbulent kinetic energy (TKE),

ii) $\nu_{t}$ is an eddy viscosity.

In order to close the system, the eddy viscosity remains to be modeled. To do so, many options are avaible (see in [4, 10, 11, 24, 26, 30, 40, 50]).

One of the most popular model is the Smagorinsky's model (see for instance in [20, 21, 24, $34,44,48,50,51,52])$, in which

$$
\nu_{t}=C_{s} \delta^{2}|D \mathbf{v}|,
$$

where $C_{s} \approx 0.1$ or 0.2 is an universal dimensionless constant, and $\delta$ a characteristic scale, ideally the size of the smallest eddies in the flow the model is supposed to catch. This model is the fundation of the wide class of Large Eddy Simulation models. The reader will find various mathematical results concerning the Smagorinsky's model in [3, 11, 24, 35, 43]. 
We next mention the so-called TKE model, given by

$$
\nu_{t}=C_{k} \ell \sqrt{k}
$$

which gives accurate results for the simulation of realistic flows (see for instance [33]). In model (3.10), $\ell$ denotes the Prandtl mixing length, $C_{k}$ is a dimensionless constant that must be fixed according to experimental data. In practice, $\ell$ is taken to be equal to the local mesh size in a numerical simulation, and $k$ is computed by using the closure equation (see in $[11,40]$ )

$$
\partial_{t} k+\overline{\mathbf{v}} \cdot \nabla k-\nabla \cdot\left(\nu_{t} \nabla k\right)=\nu_{t}|D \overline{\mathbf{v}}|^{2}-\frac{k \sqrt{k}}{\ell} .
$$

The reader will find a bunch of mathematical result concerning the coupling of the TKE equation to the mean NSE in [7, 8, 11, 19, 27, 30].

Finally, we mention the famous $k-\mathscr{E}$ model that is used for the numerical simulations carried out in Section 5. In this model, $\mathscr{E}$ denotes the turbulent dissipation

$$
\mathscr{E}=2 \nu \overline{\left|D \mathbf{v}^{\prime}\right|^{2}}
$$

and dimensional analysis leads to write

$$
\nu_{t}=C_{\mu} \frac{k^{2}}{\mathscr{E}} .
$$

The coupled system used to compute $k$ and $\mathscr{E}$ is the following (see [11, 40] for the derivation of these equations):

$$
\left\{\begin{aligned}
\partial_{t} k+\overline{\mathbf{v}} \cdot \nabla k-\nabla \cdot\left(\nu_{t} \nabla k\right) & =\nu_{t}|D \overline{\mathbf{v}}|^{2}-\mathscr{E} \\
\partial_{t} \mathscr{E}+\overline{\mathbf{v}} \cdot \nabla \mathscr{E}-\nabla \cdot\left(\nu_{t} \nabla \mathscr{E}\right) & =c_{\eta} k|D \overline{\mathbf{v}}|^{2}-c_{\mathscr{E}} \frac{\mathscr{E}^{2}}{k},
\end{aligned}\right.
$$

where $C_{\nu}=0.09, c_{\mathscr{E}}=1.92$ and $c_{\eta}=1.44$ are dimensionless constants.

\section{Law of the $-5 / 3$}

The idea behind the law of the $-5 / 3$ for homogeneous and isotropic turbulence is that in the « inertial range », the energy density $E=E(k)$ at a given point $(t, \mathbf{x})$ is driven by the dissipation $\mathscr{E}$. In this section, we properly define the energy density $E$ for homogeneous and isotropic turbulent flows. We then set the frame of the dimensional bases and the similarity principle in order to rigorously derive the law of the $-5 / 3$.

Remark 4.1. For homogeneous and isotropic turbulence, one can show the identity $\mathscr{E}=$ $2 \nu \overline{\left|D \mathbf{v}^{\prime}\right|^{2}}=2 \nu \overline{|D \mathbf{v}|^{2}}$ (see in [11]).

\subsection{Energy density of the flow}

Roughly speaking, homogeneity and isotropy means that the correlations in the flows are invariant under translations and isometries (see in [2, 11, 32]), which we assume throughout this section, as well as the stationarity of the mean flow for simplicity. Let

$$
\mathbb{E}=\frac{1}{2} \overline{|\mathbf{v}|^{2}}
$$

be the total mean kinetic energy at a given point $\mathbf{x} \in \Omega$, which we not specify in what follows. 
Theorem 4.1. There exists a measurable function $E=E(k)$, defined over $\mathbb{R}_{+}$, the integral of which over $\mathbb{R}_{+}$is finite, and such that

$$
\mathbb{E}=\int_{0}^{\infty} E(k) d k .
$$

Proof. Let $\mathbb{B}_{2}$ be the two order correlation tensor expressed by:

$$
\mathbb{B}_{2}=\mathbb{B}_{2}(\mathbf{r})=\left(\overline{v_{i}(\mathbf{x}) v_{j}(\mathbf{x}+\mathbf{r})}\right)_{1 \leq i, j \leq 3}=\left(B_{i j}(\mathbf{r})\right)_{1 \leq i, j \leq 3},
$$

which only depend on $\mathbf{r}$ by the homogeneity assumption, nor on $t$ because of the stationarity assumption. It is worth noting that

$$
\mathbb{E}=\frac{1}{2} \operatorname{tr} \mathbb{B}_{2}(0)
$$

Let $\widehat{\mathbb{B}}_{2}$ denotes the Fourier transform of $\mathbb{B}$ expressed by

$$
\forall \mathbf{k} \in \mathbb{R}^{3}, \quad \widehat{\mathbb{B}}_{2}(\mathbf{k})=\frac{1}{(2 \pi)^{3}} \int_{\mathbf{R}^{3}} \mathbb{B}_{2}(\mathbf{r}) e^{-i \mathbf{k} \cdot \mathbf{r}} d \mathbf{r},
$$

We deduce from the Plancherel formula,

$$
\forall \mathbf{r} \in \mathbb{R}^{3}, \quad \mathbb{B}_{2}(\mathbf{r})=\frac{1}{(2 \pi)^{3}} \int_{\mathbf{R}^{3}} \widehat{\mathbb{B}}_{2}(\mathbf{k}) e^{i \mathbf{k} \cdot \mathbf{r}} d \mathbf{k},
$$

which makes sense for both types of solutions to the NSE, strong or turbulent (see the section 2). It is easily checked that the isotrpoy of $\mathbb{B}_{2}$ in $\mathbf{r}$ yields the isotropy of $\widehat{\mathbb{B}}_{2}$ in k. Therefore, according to Theorem 5.1 in [11] we deduce the existence of two real valued functions $\widetilde{B}_{d}$ and $\widetilde{B}_{n}$ of class $C^{1}$ such that ${ }^{1}$

$$
\forall \mathbf{k} \in \mathbb{R}^{3}, \quad|\mathbf{k}|=k, \quad \widehat{\mathbb{B}}_{2}(\mathbf{k})=\left(\widetilde{B}_{d}(k)-\widetilde{B}_{n}(k)\right) \frac{\mathbf{k} \otimes \mathbf{k}}{k^{2}}+\widetilde{B}_{n}(k) \mathrm{I}_{3} .
$$

Using formula (4.7) yields

$$
\widehat{B}_{i i}(\mathbf{k})=\widetilde{B}_{d}(k)+2 \widetilde{B}_{n}(k),
$$

which combined with Fubini's Theorem, (4.4) and (4.6), leads to

$$
\int_{\mathbf{R}^{3}} \widehat{B}_{i i}(\mathbf{k}) d \mathbf{k}=\int_{0}^{\infty}\left(\int_{|\mathbf{k}|=k} \widehat{B}_{i i}(\mathbf{k}) d \boldsymbol{\sigma}\right) d k=\int_{0}^{\infty} 4 \pi k^{2}\left(\widetilde{B}_{d}(k)+2 \widetilde{B}_{n}(k)\right) d k,
$$

by noting $d \boldsymbol{\sigma}$ the standard measure over the sphere $\{|\mathbf{k}|=k\}$. This proves the result, where $E(k)$ is given by

$$
E(k)=\left(\frac{k}{2 \pi}\right)^{2}\left(\widetilde{B}_{d}(k)+2 \widetilde{B}_{n}(k)\right)
$$

Remark 4.2. From the physical point of view, $E(k)$ is the amount of kinetic energy in the sphere $S_{k}=\{|\mathbf{k}|=k\}$. As such, it is expected that $E \geq 0$ in $\mathbb{R}$, and we deduce from (4.2) that $E \in L^{1}\left(\mathbb{R}_{+}\right)$. Unfortunately, we are not able to prove that $E \geq 0$ from formula (4.10), which remains an open problem.

\footnotetext{
${ }^{1} k$ already denotes the TKE, and from now also the wavenumber, $k=|\mathbf{k}|$. This is commonly used in turbulence modeling, although it might sometimes be confusing.
} 


\subsection{Dimensional bases}

Only length and time are involved in this frame, since we do not consider heat transfers and the fluid is incompressible. Therefore, any field $\psi$ related to the flow has a dimension $[\psi]$ encoded as:

$$
[\psi]=(\text { length })^{d_{\ell}(\psi)}(\text { time })^{d_{\tau}(\psi)},
$$

which we express through the couple

$$
\mathbb{D}(\psi)=\left(d_{\ell}(\psi), d_{\tau}(\psi)\right) \in \mathbb{Q}^{2} .
$$

Definition 4.1. A length-time basis is a couple $b=(\lambda, \tau)$, where $\lambda$ is a given constant length and $\tau$ a constant time.

Definition 4.2. Let $\psi=\psi(t, \mathbf{x})$ (constant, scalar, vector, tensor...) be defined on $Q=$ $[0, T] \times \Omega$. Let $\psi_{b}$ be the dimensionless field defined by:

$$
\psi_{b}\left(t^{\prime}, \mathbf{x}^{\prime}\right)=\lambda^{-d_{\ell}(\psi)} \tau^{-d_{\tau}(\psi)} \psi\left(\tau t^{\prime}, \lambda \mathbf{x}^{\prime}\right),
$$

where

$$
\left(t^{\prime}, \mathbf{x}^{\prime}\right) \in Q_{b}=\left[0, \frac{T}{\tau}\right] \times \frac{1}{\lambda} \Omega
$$

is dimensionless. We say that $\psi_{b}=\psi_{b}\left(t^{\prime}, \mathbf{x}^{\prime}\right)$ is the b-dimensionless field deduced from $\psi$.

\subsection{Kolmogorov scales}

Let us consider the length-time basis $b_{0}=\left(\lambda_{0}, \tau_{0}\right)$, given by

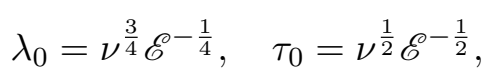

where $\mathscr{E}$ is the dissipation defined by (3.12) (see also Remark 4.1). The scale $\lambda_{0}$ is kown as the Kolmogorov scale. The important point here is that

$$
\mathscr{E}_{b_{0}}=\nu_{b_{0}}=1 .
$$

Moreover, for all wave number $k$, and because

$$
\mathbb{D}(E)=(3,-2),
$$

we get

$$
E(k)=\lambda_{0}^{3} \tau_{0}^{-2} E_{b_{0}}\left(\lambda_{0} k\right)=\nu^{\frac{5}{4}} \mathscr{E}^{\frac{1}{4}} E_{b_{0}}\left(\lambda_{0} k\right),
$$

by using (4.13). We must determine the universal profil $E_{b_{0}}$.

\subsection{Proof of the $-5 / 3$ 's law}

The law of the $-5 / 3$ is based on two assumptions about the flow:

i) the separation of the scales (assumption 4.1 below),

ii) the similarity assumption (assumption 4.2 below). 
Assumption 4.1. Let $\ell$ be the Prandtl mixing length. Then

$$
\lambda_{0}<<\ell \text {. }
$$

Assumption 4.2. There exists an interval

$$
\begin{gathered}
{\left[k_{1}, k_{2}\right] \subset\left[\frac{2 \pi}{\ell}, \frac{2 \pi}{\lambda_{0}}\right] \text { s.t. } k_{1}<<k_{2} \text { and on }\left[\lambda_{0} k_{1}, \lambda_{0} k_{2}\right],} \\
\forall b_{1}=\left(\lambda_{1}, \tau_{1}\right), b_{2}=\left(\lambda_{2}, \tau_{2}\right) \text { s.t. } \mathscr{E}_{b_{1}}=\mathscr{E}_{b_{2}} \text {, then } E_{b_{1}}=E_{b_{2}} .
\end{gathered}
$$

Theorem 4.2. Scale separation and similarity assumptions 4.1 and 4.2 yield the existence of a constant $C$ such that

$$
\forall k^{\prime} \in\left[\lambda_{0} k_{1}, \lambda_{0} k_{2}\right]=J_{r}, \quad E_{b_{0}}\left(k^{\prime}\right)=C\left(k^{\prime}\right)^{-\frac{5}{3}} .
$$

Corollary 4.1. The energy spectrum satisfies the $-5 / 3$ law

$$
\forall k \in\left[k_{1}, k_{2}\right], \quad E(k)=C \mathscr{E}^{\frac{2}{3}} k^{-\frac{5}{3}},
$$

where $C$ is a dimensionless constant.

Proof. Let

$$
b^{(\alpha)}=\left(\alpha^{3} \lambda_{0}, \alpha^{2} \tau_{0}\right) .
$$

As

$$
\mathscr{E}_{b^{(\alpha)}}=1=\mathscr{E}_{b_{0}}
$$

the similarity assumption yields

$$
\forall k^{\prime} \in J_{r}, \quad \forall \alpha>0, \quad E_{b^{(\alpha)}}\left(k^{\prime}\right)=E_{b_{0}}\left(k^{\prime}\right),
$$

which leads to the functional equation,

$$
\forall k^{\prime} \in J_{r}, \quad \forall \alpha>0, \quad \frac{1}{\alpha^{5}} E_{b_{0}}\left(k^{\prime}\right)=E_{b_{0}}\left(\alpha^{3} k^{\prime}\right),
$$

whose unique solution is given by

$$
\forall k^{\prime} \in J_{r}, \quad E_{b_{0}}\left(k^{\prime}\right)=C\left(k^{\prime}\right)^{-\frac{5}{3}}, \quad C=\left(\frac{k_{1}}{\lambda_{0}}\right)^{\frac{5}{3}} E_{0}\left(\frac{k_{1}}{\lambda_{0}}\right),
$$

hence the result. Corollary 4.1 is a direct consequence of (4.16) combined with (4.19).

Remark 4.3. It can be shown that the law of $-5 / 3$ yields the Smagorinsky's model (3.9) (see in [11]). 


\section{$5 \quad$ Numerical experiments}

\subsection{Simulation setting}

The computational domain $\Omega$ is a box, the size $L_{x} \times L_{y} \times L_{z}$ of which is equal to $(1024 m, 512 m, 200 m)$ (see figure 3$)$. The number of nodes is $(256,128,64)$. The bottom of the box, plotted in figure 3 , has a non trivial topography modeled by gaussian smooth domes, the height of which being equal to $50 \mathrm{~m}$. We perform the simulation with $\nu=2.10^{-5} \mathrm{~m}^{2} \mathrm{~s}^{-1}$, which yields a Reynolds number equal to $9.10^{7}$. We use the mean NSE with the Boussinesq assumption, coupled to the $k-\mathscr{E}$ model, namely the PDE system (3.6)(3.8)-(3.13)-(3.14). We specify in what follows the boundary conditions, by considering the following decomposition of $\Gamma=\partial \Omega$ :

$$
\Gamma=\Gamma_{t} \cup \Gamma_{f} \cup \Gamma_{b} \cup \Gamma_{g} \cup \Gamma_{i} \cup \Gamma_{o},
$$

where

- $\Gamma_{t}$ is the top of the box,

- $\Gamma_{f}$ is the front face,

- $\Gamma_{b}$ is the back face,

- $\Gamma_{g}$ is the bottom of the box (the ground),

- $\Gamma_{i}$ is the inlet,

- $\Gamma_{o}$ is the outlet.

The condition on $\Gamma_{i}$ is prescribed by the Monin Obukhov similitude law [41]:

$$
\left.\mathbf{v}(x, y, z, t)\right|_{\Gamma_{i}}=\left(\frac{u_{\star}}{\kappa} \ln \left(\frac{z+z_{0}}{z_{0}}\right), 0,0\right)^{t},
$$

where $\kappa=0.4$ is the Von Karman constant, $z$ denotes the distance from the ground level, the aerodynamic roughness length $z_{0}$ is equal to $0.1 \mathrm{~m}$, the friction velocity is expressd by:

$$
u_{\star}=\kappa U_{r e f}\left[\ln \left(\frac{H_{r e f}+z_{0}}{z_{0}}\right)\right]^{-1},
$$

by taking $U_{r e f}=36 \mathrm{~ms}^{-1}$ and $H_{r e f}=200 \mathrm{~m}$. The turbulent kinetic energy and turbulent dissipation are setted by

$$
\left\{\begin{array}{l}
\left.k\right|_{\Gamma_{i}}=u_{\star}^{1 / 2} C_{\nu}^{-1 / 2} \\
\left.\mathscr{E}\right|_{\Gamma_{i}}=\frac{u_{\star}^{3}}{\kappa\left(z+z_{0}\right)} .
\end{array}\right.
$$

On $\Gamma_{b}$, velocity, TKE and turbulent dissipation are subject to verify the no slip and homogeneous boundary conditions,

$$
\begin{cases}\left.\mathbf{v}\right|_{\Gamma_{g}} & =(0,0,0)^{t} \\ \left.k\right|_{\Gamma_{g}} & =0 \\ \left.\mathscr{E}\right|_{\Gamma_{g}} & =0\end{cases}
$$


On the top and lateral boundaries, we put

$$
\left\{\begin{array}{llll}
\mathbf{v} \cdot \mathbf{n} & =0 & \text { on } & \Gamma_{t} \cup \Gamma_{b} \cup \Gamma_{f}, \\
\nabla k \cdot \mathbf{n}=0 & \text { on } & \Gamma_{t} \cup \Gamma_{b} \cup \Gamma_{f}, \\
\nabla \mathscr{E} \cdot \mathbf{n}=0 & \text { on } & \Gamma_{t} \cup \Gamma_{b} \cup \Gamma_{f} .
\end{array}\right.
$$

Finally a null gradient condition is prescribed at the outlet $\Gamma_{o}$

$$
\left\{\begin{array}{llll}
\nabla(\mathbf{v} \cdot \mathbf{n}) & =0 & \text { on } & \Gamma_{o} \\
\nabla k \cdot \mathbf{n} & =0 & \text { on } & \Gamma_{o}, \\
\nabla \mathscr{E} \cdot \mathbf{n} & =0 & \text { on } & \Gamma_{o} .
\end{array}\right.
$$

Remark 5.1. The PDE system (3.6)-(3.8)-(3.13)-(3.14) with the boundary conditions (5.1)-(5.3)-(5.4)-(5.5)-(5.6) yields a very hard mathematical problem. The existence and the uniqueness of a solution is a difficult issue, whether for global weak solutions or local time strong solutions.

\subsection{Results}

The numerical scheme we use for the simulation is based on the standard finite volume method (FVM) in space, and a Euler method for the time discretization. For the simplicity, we will not write here this technical part of the work. The reader will find comprehensive presentations of the FVM in [15, 17, 23, 57].

The simulation reaches a statistical equilibrium in about 180 physical seconds, which is the time at which the results are displayed. In figures 4 and 5, are plotted the values of the stramwise and spanwise components of the velocity at $z=50 \mathrm{~m}$, which corresponds to the dome height.

In Figure 6, we have plotted the energy spectrum of the flow at $(x, y, z)=(500,200,50)$ using a log-log scale, together with a straight line whose slope is equal to $-5 / 3=-1,666 \ldots$. and the regression straight line of $\log _{10}(E(k))$, whose slope is about equal to -2.1424 . The results call for the following comments.

i) The simulation reveals a certain reliability of the code, which suggests the convergence of the numerical method. However, the mathematical convergence of the sheme remains an open question, closely related to the question of the existence of solutions mentionned in Remark 5.1.

ii) The curve $\log _{10}(E(k))$ is an irregular curve which substancially differs from a straight line, so that we cannot conclude that numerically $E(k)$ behaves like $C^{t e} k^{\alpha}$ in some interval $\left[k_{1}, k_{2}\right]$. Moreover, there is a gap between the slope of the regression straight line of the curve and $-5 / 3$. However, something that looks like an inertial range can be identified between $k=10^{-5} m^{-1}$ and $k=10^{-1} m^{-1}$. This departure from the $-5 / 3$ law asks for the following comments and questions.

- The case under consideration yields a turbulence which is not homogeneous nor isotropic, which may explain the slope equal to -2.1424 we found.

- This simulation does not validate the Kolmogorov law or any law like $E(k) \approx$ $C^{t e} k^{\alpha}$. We cannot infer that such a law holds or not. Many parameters may generate the oscillations we observe in the curve $\log _{10}(E(k))$, such as any eventual numerical dissipation, a wrong choice of the constants in the $k-\mathscr{E}$ model which also may be not accurate, the boundary conditions we used and which may be questionable. 


\section{References}

[1] David. Apsley and Ian. Castro. A limited-length-scale k-epsilon model for the neutral and stably-stratified atmospheric boundary layer. Boundary-Layer Meteorology, 83(1):75-98, 1997.

[2] George K. Batchelor. The theory of homogeneous turbulence. Cambridge Monographs on Mechanics and Applied Mathematics. Cambridge University Press, New York, 1959.

[3] H. Beirão da Veiga. On the Ladyzhenskaya-Smagorinsky turbulence model of the Navier-Stokes equations in smooth domains. The regularity problem. J. Eur. Math. Soc. (JEMS), 11(1):127-167, 2009.

[4] L. C. Berselli, T. Iliescu, and W. J. Layton. Mathematics of large eddy simulation of turbulent flows. Scientific Computation. Springer-Verlag, Berlin, 2006.

[5] Elie Bou-Zeid. Large-eddy simulation of neutral atmospheric boundary layer flow over heterogeneous surfaces: Blending height and effective surface roughness. Water Resources Research, 40(2):1-18, 2004.

[6] Joseph Boussinesq. Essai sur la théorie des eaux courantes. Mémoires présentés par divers savants à l'Académie des Sciences, 23(1):1-660, 1877.

[7] Françoise Brossier and Roger Lewandowski. Impact of the variations of the mixing length in a first order turbulent closure system. M2AN Math. Model. Numer. Anal., 36(2):345-372, 2002.

[8] Miroslav Bulíček, Roger Lewandowski, and Josef Málek. On evolutionary NavierStokes-Fourier type systems in three spatial dimensions. Comment. Math. Univ. Carolin., 52(1):89-114, 2011.

[9] Marco Cannone. Harmonic analysis tools for solving the incompressible Navier-Stokes equations. In Handbook of mathematical fluid dynamics. Vol. III, pages 161-244. North-Holland, Amsterdam, 2004.

[10] Tomás Chacón-Rebollo and Roger Lewandowski. A variational finite element model for large-eddy simulations of turbulent flows. Chin. Ann. Math. Ser. B, 34(5):667-682, 2013.

[11] Tomás Chacón Rebollo and Roger Lewandowski. Mathematical and numerical foundations of turbulence models and applications. Modeling and Simulation in Science, Engineering and Technology. Birkhäuser/Springer, New York, 2014.

[12] Jean-Yves Chemin and Isabelle Gallagher. Wellposedness and stability results for the Navier-Stokes equations in $\mathbf{R}^{3}$. Ann. Inst. H. Poincaré Anal. Non Linéaire, 26(2):599$624,2009$.

[13] Fotini Katodopes Chow, Robert L. Street, Ming Xue, and Joel H Ferziger. Explicit filtering and reconstruction turbulence modeling for large-eddy simulation of neutral boundary layer flow. Journal of the Atmospheric Sciences, 62:2058-2077, 2005.

[14] Peter Constantin and Ciprian Foias. Navier-Stokes equations. Chicago Lectures in Mathematics. University of Chicago Press, Chicago, IL, 1988. 
[15] Robert Eymard, Thierry Gallouet, and Raphaele Herbin. Finite volume methods. In Solution of Equation in Rn (Part 3), Techniques of Scientific Computing (Part 3), volume 7 of Handbook of Numerical Analysis, pages 713 - 1018. Elsevier, 2000.

[16] Eduard Feireisl. Dynamics of viscous compressible fluids, volume 26 of Oxford Lecture Series in Mathematics and its Applications. Oxford University Press, Oxford, 2004.

[17] Joel H Ferziger and Milovan Peric. Computational methods for fluid dynamics. Springer Science \& Business Media, 2012.

[18] Hiroshi Fujita and Tosio Kato. On the navier-stokes initial value problem. i. Archive for Rational Mechanics and Analysis, 16(4):269-315, 1964.

[19] T. Gallouët, J. Lederer, R. Lewandowski, F. Murat, and L. Tartar. On a turbulent system with unbounded eddy viscosities. Nonlinear Anal., 52(4):1051-1068, 2003.

[20] M. Germano, U. Piomelli, P. Moin, and W. Cabot. A dynamic subgrid-scale eddy viscosity model. Physics of Fluids A, 3(7):1760-1765, 1991.

[21] Massimo Germano. Fundamentals of large eddy simulation. In Advanced turbulent flow computations (Udine, 1998), volume 395 of CISM Courses and Lectures, pages 81-130. Springer, Vienna, 2000.

[22] Eberhard Hopf. uber die anfangswertaufgabe fr die hydrodynamischen grundgleichungen. Math. Nachr., 1951.

[23] H Jasak. Error analysis and estimation for the finite volume method with applications to fluid flows. 1996.

[24] Volker John. Large eddy simulation of turbulent incompressible flows, volume 34 of Lecture Notes in Computational Science and Engineering. Springer-Verlag, Berlin, 2004. Analytical and numerical results for a class of LES models.

[25] Andrej N. Kolmogorov. The Local Structure of Turbulence in Incompressible Viscous Fluid for Very Large Reynolds' Numbers. Akademiia Nauk SSSR Doklady, 30:301-305, 1941.

[26] B.E. Launder and D.B. Spalding. The numerical computation of turbulent flows. Computer Methods in Applied Mechanics and Engineering, 3(2):269-289, 1974.

[27] J. Lederer and R. Lewandowski. A RANS 3D model with unbounded eddy viscosities. Ann. Inst. H. Poincaré Anal. Non Linéaire, 24(3):413-441, 2007.

[28] Pierre-Gilles Lemarie-Rieusset. Recent developments in the Navier-Stokes problem, volume 431 of Chapman 85 Hall/CRC Research Notes in Mathematics. Chapman \& Hall/CRC, Boca Raton, FL, 2002.

[29] Jean Leray. Sur le mouvement d'un liquide visqueux emplissant l'espace. Acta Mathematica, 63(1):193-248, 1934.

[30] Roger Lewandowski. The mathematical analysis of the coupling of a turbulent kinetic energy equation to the Navier-Stokes equation with an eddy viscosity. Nonlinear Anal., 28(2):393-417, 1997. 
[31] Roger Lewandowski. Long-time turbulence model deduced from the Navier-Stokes equations. Chin. Ann. Math. Ser. B, 36(5):883-894, 2015.

[32] Roger Lewandowski. The Kolmogorov-Taylor Law of turbulence : what can rigorously be proved? Handbook of applications of chaos theory. Taylor and Francis, 2016.

[33] Roger Lewandowski and Géraldine Pichot. Numerical simulation of water flow around a rigid fishing net. Comput. Methods Appl. Mech. Engrg., 196(45-48):4737-4754, 2007.

[34] Douglas K. Lilly. Numerical simulation and prediction of atmospheric convection. In Mécanique des fluides numérique (Les Houches, 1993), pages 325-374. North-Holland, Amsterdam, 1996.

[35] J.-L. Lions. Quelques méthodes de résolution des problèmes aux limites non linéaires. Dunod; Gauthier-Villars, Paris, 1969.

[36] Pierre-Louis Lions. Mathematical topics in fluid mechanics. Vol. 1, volume 3 of $O x$ ford Lecture Series in Mathematics and its Applications. The Clarendon Press, Oxford University Press, New York, 1996. Incompressible models, Oxford Science Publications.

[37] Nikola Marjanovic, Sonia Wharton, and Fotini K. Chow. Investigation of model parameters for high-resolution wind energy forecasting: Case studies over simple and complex terrain. Journal of Wind Engineering and Industrial Aerodynamics, 134:10$24,2014$.

[38] P.J Mason. Large-eddy simulation of the convective atmospheric boundary layer. $J$. Atmos. Sci., 1989.

[39] T. Michioka, a. Sato, and K. Sada. Large-eddy simulation coupled to mesoscale meteorological model for gas dispersion in an urban district. Atmospheric Environment, $75(\mathrm{x}): 153-162,2013$.

[40] B. Mohammadi and O. Pironneau. Analysis of the k-epsilon turbulence model. RAM: Research in Applied Mathematics. Masson, Paris; John Wiley \& Sons, Ltd., Chichester, 1994.

[41] a.S. Monin and a.M. Obukhov. Basic laws of turbulent mixing in the surface layer of the atmosphere. Contrib. Geophys. Inst. Acad. Sci. USSR, 24(151):163-187, 1954.

[42] L. Onsager. Statistical hydrodynamics. Nuovo Cimento (9), 6(Supplemento, 2(Convegno Internazionale di Meccanica Statistica)):279-287, 1949.

[43] Carlos Pares Madroñal. Étude mathématique et approximation numérique de quelques problèmes aux limites de la mécanique des fluides incompressibles. Institut National de Recherche en Informatique et en Automatique (INRIA), Rocquencourt, 1992.

[44] Stephen B. Pope. Turbulent flows. Cambridge University Press, Cambridge, 2000.

[45] Fernando Porté-Agel, Charles Meneveau, and Marc B Parlange. A scale-dependent dynamic model for large-eddy simulation: application to a neutral atmospheric boundary layer. Journal of Fluid Mechanics, 415:261-284, 2000. 
[46] Fernando PortÂl'-Agel, Yu-Ting Wu, Hao Lu, and Robert J. Conzemius. Large-eddy simulation of atmospheric boundary layer flow through wind turbines and wind farms. Journal of Wind Engineering and Industrial Aerodynamics, 99(4):154-168, 2011. The Fifth International Symposium on Computational Wind Engineering.

[47] Ludwig Prandtl. über die ausgebildeten turbulenz. Zeitschrift für angewandte Mathematik und Mechanik, 5:136-139, 1925.

[48] Ludwig Prandtl. Prandtl-Essentials of fluid mechanics, volume 158 of Applied Mathematical Sciences. Springer, New York, third edition, 2010. Translated from the 12th German edition by Katherine Asfaw and edited by Herbert Oertel, With contributions by P. Erhard, D. Etling, U. Müller, U. Riedel, K. R. Sreenivasan and J. Warnatz.

[49] Osborne Reynolds. An experimental investigation of the circumstances which determine whether the motion of water shall be direct or sinuous, and of the law of resistance in parallel channels. Philosophical Transactions of the Royal Society, 174:935-982, 1883.

[50] Pierre Sagaut. Large eddy simulation for incompressible flows. Scientific Computation. Springer-Verlag, Berlin, third edition, 2006. An introduction, Translated from the 1998 French original, With forewords by Marcel Lesieur and Massimo Germano, With a foreword by Charles Meneveau.

[51] Joseph Smagorinsky. On the application of numerical methods to the solution of systems of partial differential equations arising in meteorology. In Frontiers of numerical mathematics, pages 107-125. University of Wisconsin Press, Madison, Wis., 1960.

[52] Joseph Smagorinsky. General circulation experiments with the primitive equations. Monthly Weather Review, 93(3):99, 1963.

[53] George G. Stokes. On the effect of the internal friction of fluids on the motion of pendulums. Transactions of the Cambridge Philosophical Society, 9:8-106, 1851).

[54] Geoffrey Ingram Taylor. Statistical theory of turbulence. part i-iv. Proc. Roy. Soc. A., 151:421-478, 1935.

[55] Roger Temam. Navier-Stokes equations. AMS Chelsea Publishing, Providence, RI, 2001. Theory and numerical analysis, Reprint of the 1984 edition.

[56] V.M Tikhomirov. Selected Works of A.N. Kolmogorov: Volume I: Mathematics and Mechanics. Tikhomirov, V.M.(ed.) Kluwer Academic Publishers, Dordrecht, Boston, London, 1992.

[57] H. G. Weller, G. Tabor, H. Jasak, and C. Fureby. A tensorial approach to computational continuum mechanics using object-oriented techniques. Comput. Phys., 12(6):620-631, November 1998.

[58] Bowen Zhou and Fotini Katopodes Chow. Nested large-eddy simulations of the intermittently turbulent stable atmospheric boundary layer over real terrain. Journal of the Atmospheric Sciences, 71(3):1021-1039, 2014.

[59] Bowen Zhou, Jason S. Simon, and Fotini K. Chow. The convective boundary layer in the terra incognita. Journal of the Atmospheric Sciences, (10 m):2545-2563, 2014. 


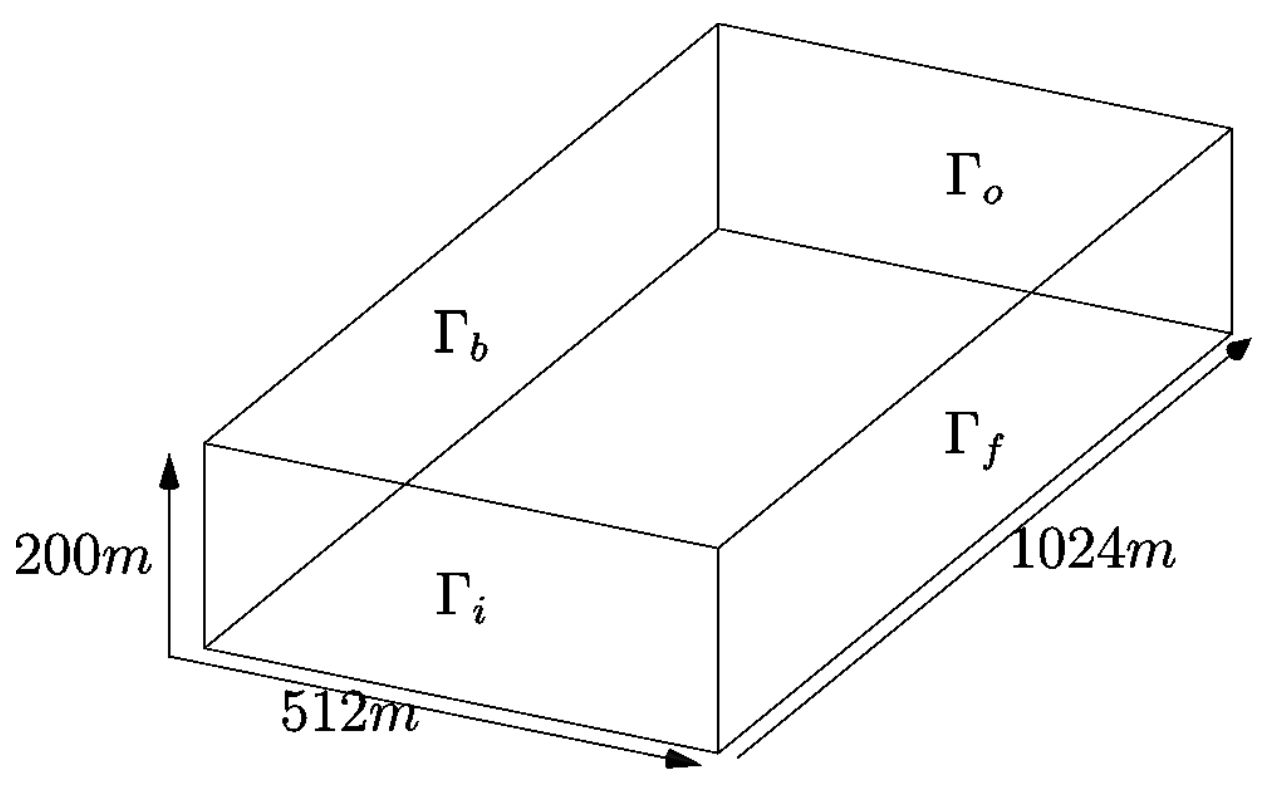

Figure 2: Computational Box 


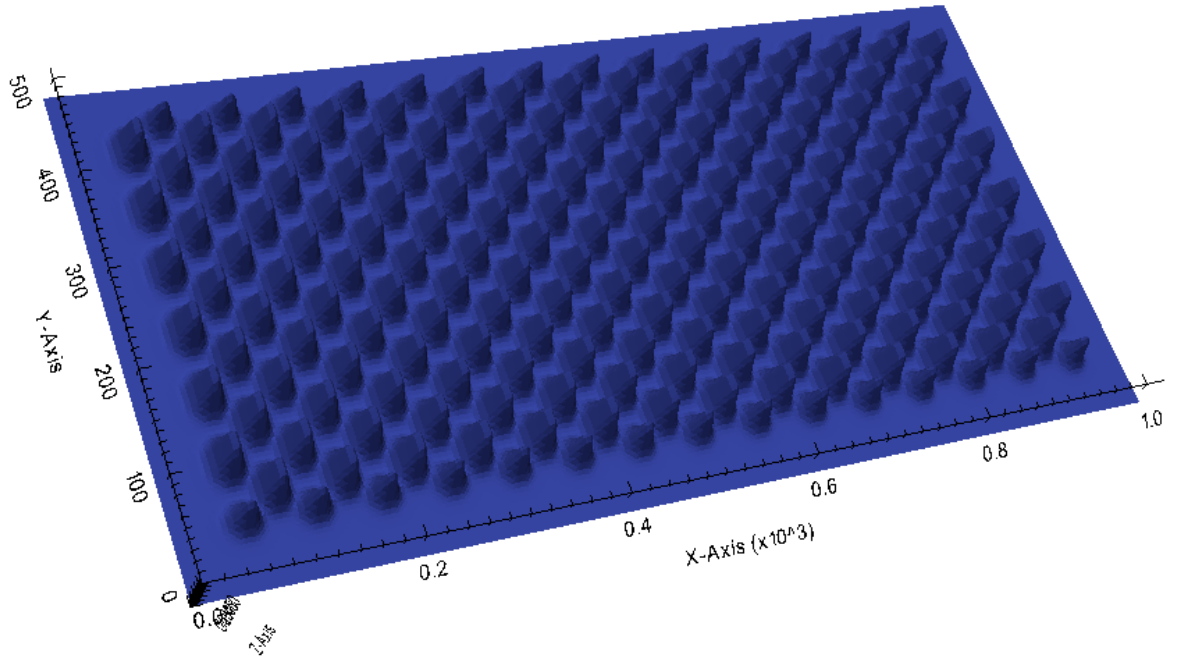

Figure 3: View of the ground.

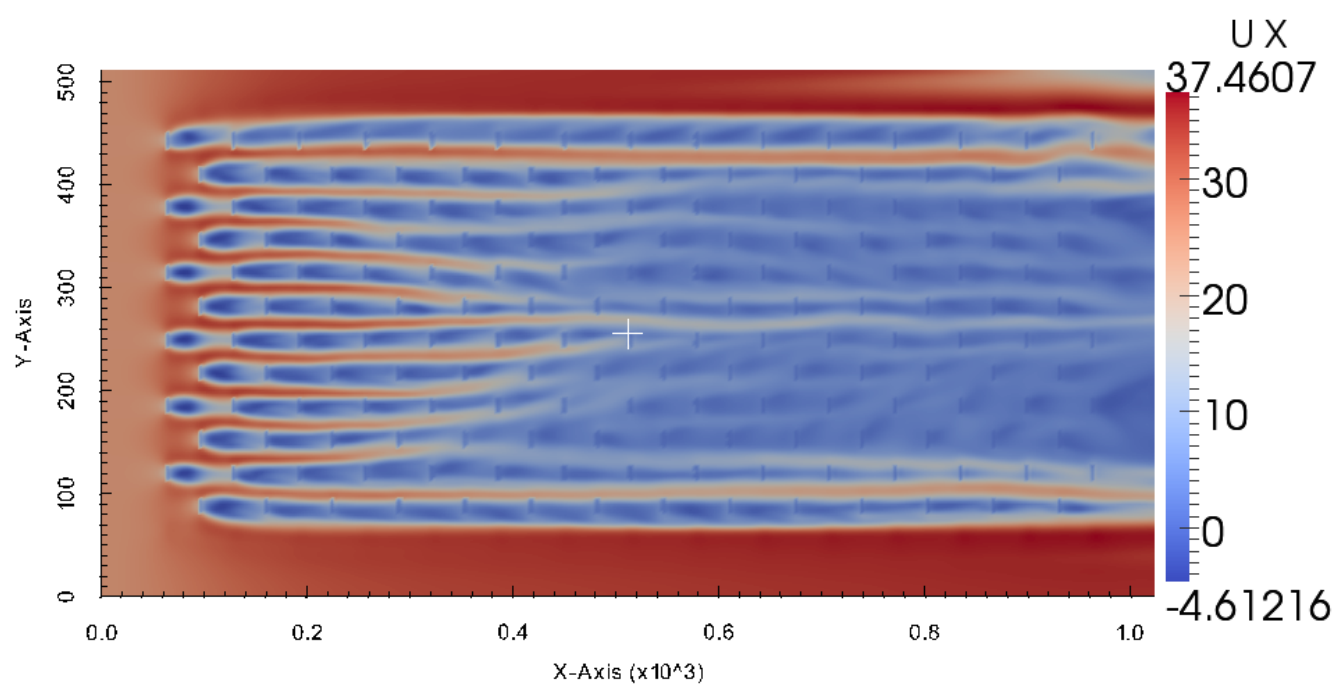

Figure 4: Streamwise direction of the flow at the $z=50 \mathrm{~m}$ cutplane. 


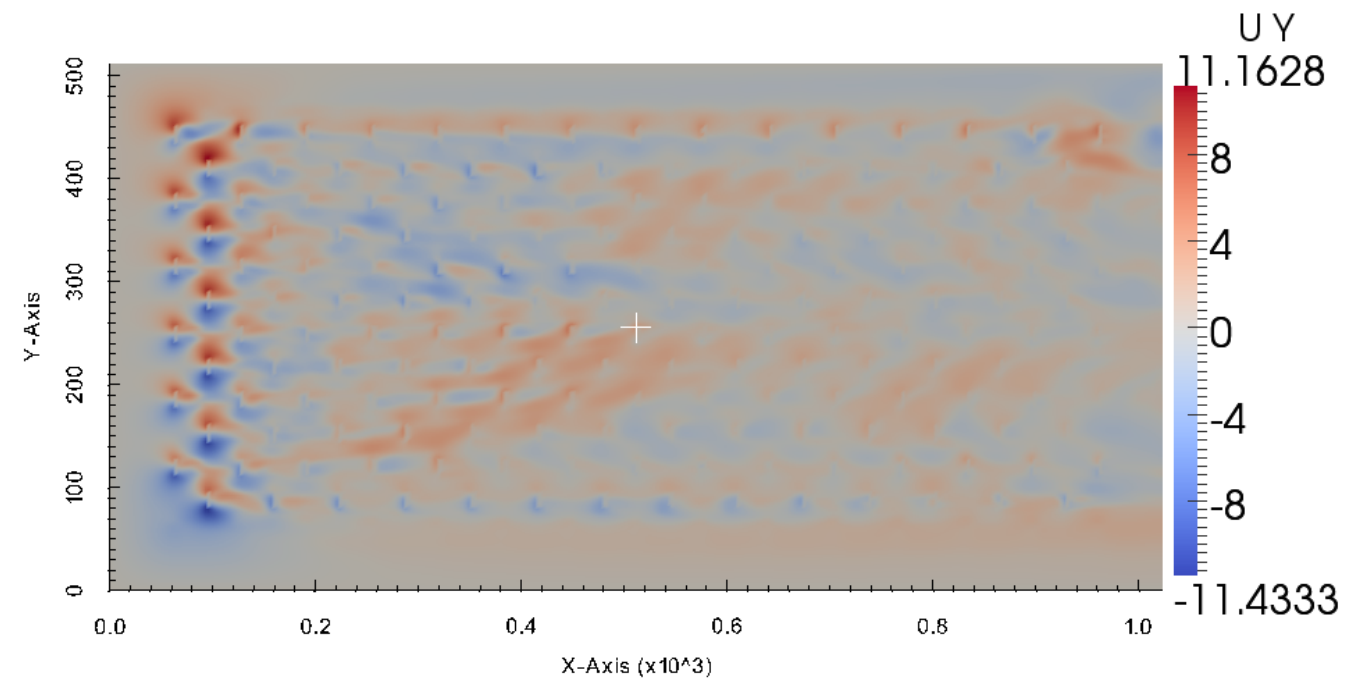

Figure 5: Spanwise direction at the $z=50 \mathrm{~m}$ cutplane 


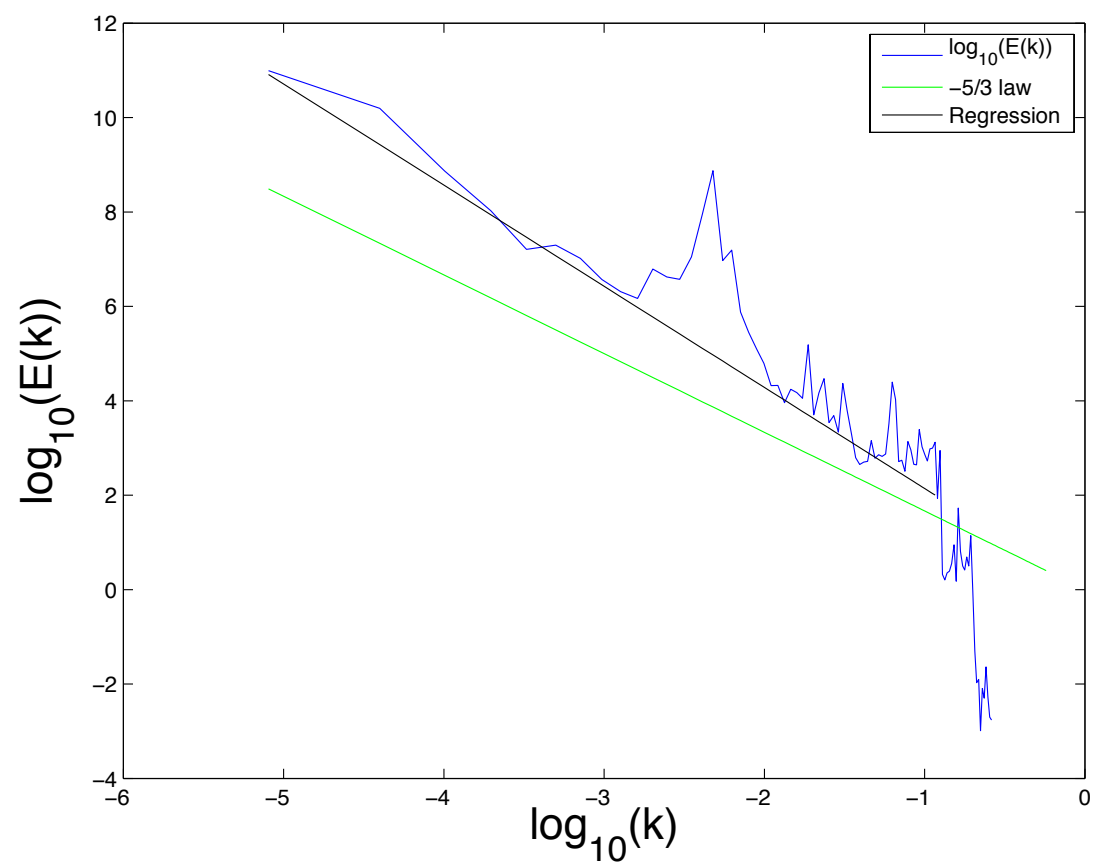

Figure 6: Energy spectrum at the point $(x, y, z)=(500,200,50)$ 